\title{
Study on the Latter Stage Evaluation to Technique Import Project
}

\author{
SHI Bao-juan ${ }^{1}$, ZHAO Guo-jie ${ }^{2}$, WANG Wei ${ }^{3}$ \\ (1. School of Management, Hebei Polytechnic University, Tangshan 063009, China; 2. School of Management, \\ Shandong University of Finance, Jinan 250014, China; 3. School of Management, Tianjin University, Tianjin 300072, China)
}

\begin{abstract}
How's the effect of technical import actually? The latter stage evaluation to technique import project is necessary to get the answer. Therefore, the latter stage evaluation to technique import project is not equal to the latter stage evaluation to general construction projects, it doesn't emphasize particularly on its economic profits but the performance of the digest, generalization, improvement and redevelopment of the imported technique.
\end{abstract}

Key words: technical import; technique import project; the latter stage evaluation

\section{Object of Study}

With the reform and opening-up, China has chosen such a technological development strategy that we take the technical import primarily and develop the technological innovation as auxiliary. How's the effect of technical import actually? The latter stage evaluation to technique import project is necessary to get the answer. This topic is significant and practical.

It seems that project's latter stage evaluation has classical system analysis method, such as the project's latter stage evaluation of World Bank. However, the latter stage evaluation to technique import project is not equal to the latter stage evaluation to general construction projects, just as the feasibility study of technique import project is not equal to the feasibility study of general construction project. Because the core or key of technique import is to digest, generalize, improve and redevelop technique.

According to the Chinese and foreign historical experience, the results of the economic backwardness country imports the foreign technique is divided into three kinds approximately:

(1) Import country's science and technique level and international advanced level maintain equidistance. For example, import country's technique level was raised when they bought some advanced technique and equipment, but it was out of time years later, so the country bought technique and equipment again in order to raise their technique level....This circle only maintain some kind of equidistance.

(2) The disparity between import country's technique level and international advanced level reduces gradually. For example, in the history, England and America in the $15^{\text {th }}$ to $17^{\text {th }}$ century, and Japan after the Second World War have ever done this.

(3) The disparity between import country's technique level and international advanced level increases

SHI Bao-juan, female, Ph.D., associate professor of School of Management, Hebei Polytechnic University; research field: management science and engineering.

ZHAO Guo-jie, male, professor of School of Management, Shandong University of Finance; research field: management science and engineering.

WANG Wei, male, Ph.D. candidate of School of Management, Tianjin University; research field: management science and engineering. 
gradually. Though some developing countries import much advanced technique positively after 1950, for various reasons, the distance actually increased.

Obviously, we should try to obtain Result (2). The factor which decides whether the technique import is successful is various, but the history incontrovertibly has proven that it was impossible to achieve the second kind of result owing to the lack of digest, generalization, improvement and redevelopment of technique.

In the previous 30 years after the foundation of China, the country belongs to the first kind basically in consequence of technical import. For instance, mechanical industry in the first five-year plan period depends on the import of advanced techniques and equipments. This made our technique level increased to the close international advanced level. Thirty years later, the new factories and equipments became old, but our technique level still paused during that time, many departments have to do the technique import again. Thus it can be seen that buying a set of modernized technology and equipment can not solve the basic problem, maybe only its digestion, improvement and redevelopment can truly solve the problem.

To the digest, generalization, improvement and redevelopment of the imported technique, digest is the precondition, which make it possible for generalization, improvement and redevelopment only when the technique is digested well. Generalization is the process of digest in macroscopic significance; digest and generalization are all hotbeds of improvement and redevelopment. There may be some improvement and redevelopment in the process of digest, and there also may be some improvement and redevelopment in the process of generalization. Obviously, we must plunge powerful strength of scientific research into the digest, generalization, improvement and redevelopment of the imported technique so that we can do the work well.

Therefore, the latter stage evaluation to technique import project is not equal to the latter stage evaluation to general construction projects, it emphasizes particularly not on its economic profits but on the performance of the digest, generalization, improvement and redevelopment of the imported technique.

\section{The Classification and the Latter Stage Evaluation Framework of Technique Import Project}

The feasibility study and latter stage evaluation of technique import project in our country doesn't realize coordination yet. Therefore, the most ideal and complete improvement should start from improving the feasibility study. That is to say, the feasibility study of technique import project should include these two items:

(1) Pre-assessment of technique import project's digest and absorbing;

(2) Pre-assessment of technique import project's generalization, improvement and redevelopment.

But each enterprise, company or the higher administrative organization who has imported technique urgently needs the latter stage evaluation of the project in some kind of degree, and we must start the work before improving the feasibility study and hunt after one latter stage evaluation method which can meet our needs. This paper considers technique import project to be divided into three kinds.

First, the main purpose of import is management. Therefore the emphasis of evaluation is economic profits. It can be called management import project. For example, the second phased projects of some power plant are for generating electricity.

Second, the main purpose of import is raising technical level. So the emphasis of evaluation is technique absorbing profits. It can be called absorbency import project. Such as three processing technique import project of some power transformer equipment manufacture company. 
Third, the main purpose of import is redevelopment. Therefore the emphasis of evaluation is innovation profits. It can be called redevelopment import project.

Accordingly, the latter stage evaluation can be divided into three kinds. But in the process of evaluation, there still exists one kind of projects failed in importing. For example, generating electricity doesn't achieve the design ability, management failed consequently; or the technique doesn't be digested, absorbency is failure consequently. To these projects we can't simply consider it failed and announce its conclusion, we should carry on the further diagnosis the latter stage evaluation and find out the reason of failure. It can not only find out remedial way for itself but also be used for reference to the next import.

The latter stage evaluation framework of technique import project makes up of Figure 1.

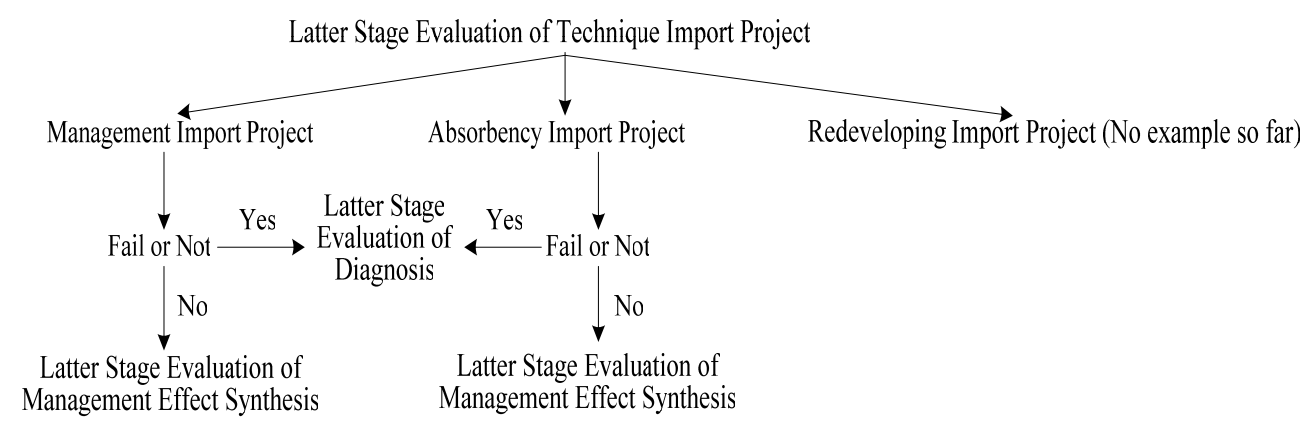

Figure 1 Latter stage evaluation framework of technique import project of some electrical power office

\section{The Latter Stage Evaluation Index System of Technique Import Project's Design and Method Choice}

Aiming at management import project, this paper chooses to design the latter stage synthetic evaluation method of management (Figure 2); aiming at absorbing import project, this paper chooses to design the latter stage synthetic evaluation method of absorbing effect (Figure 3). The main difference between them is the design of index system. As to the evaluation method, we can choose AHP or Fuzzy to establish multi-level latter stage evaluation index system of technique import project, confirm the weight and evaluate synthetically in order to make the two kinds of latter stage evaluation of technique import project systematic, scientific and applied.

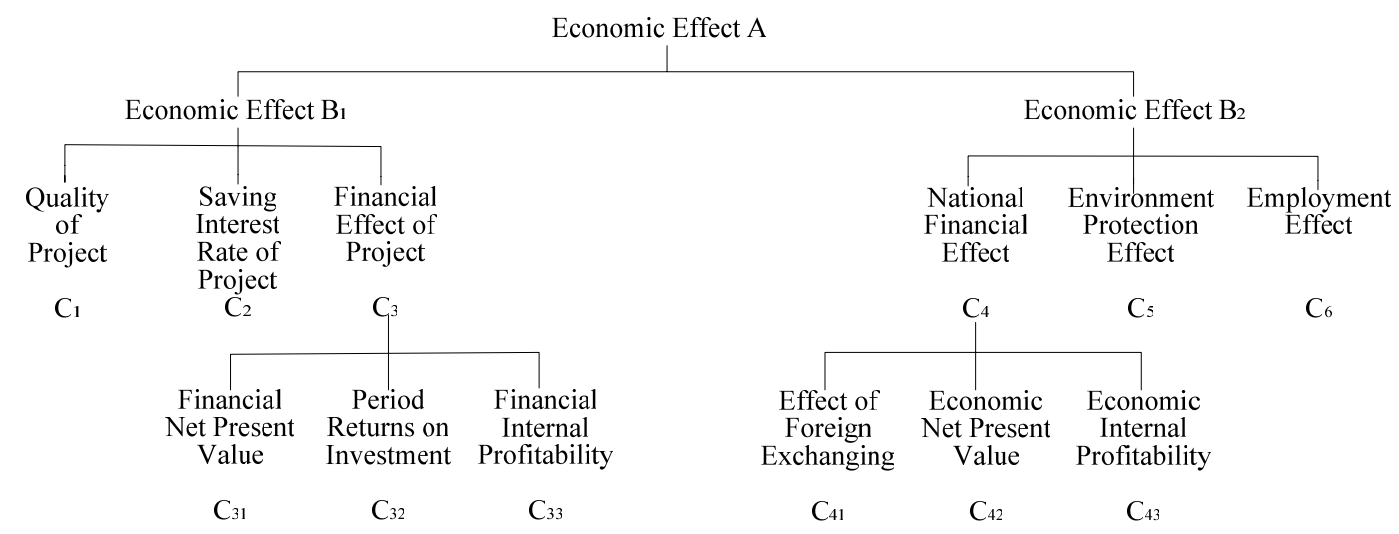

Figure 2 Latter stage evaluation index system of management import project 


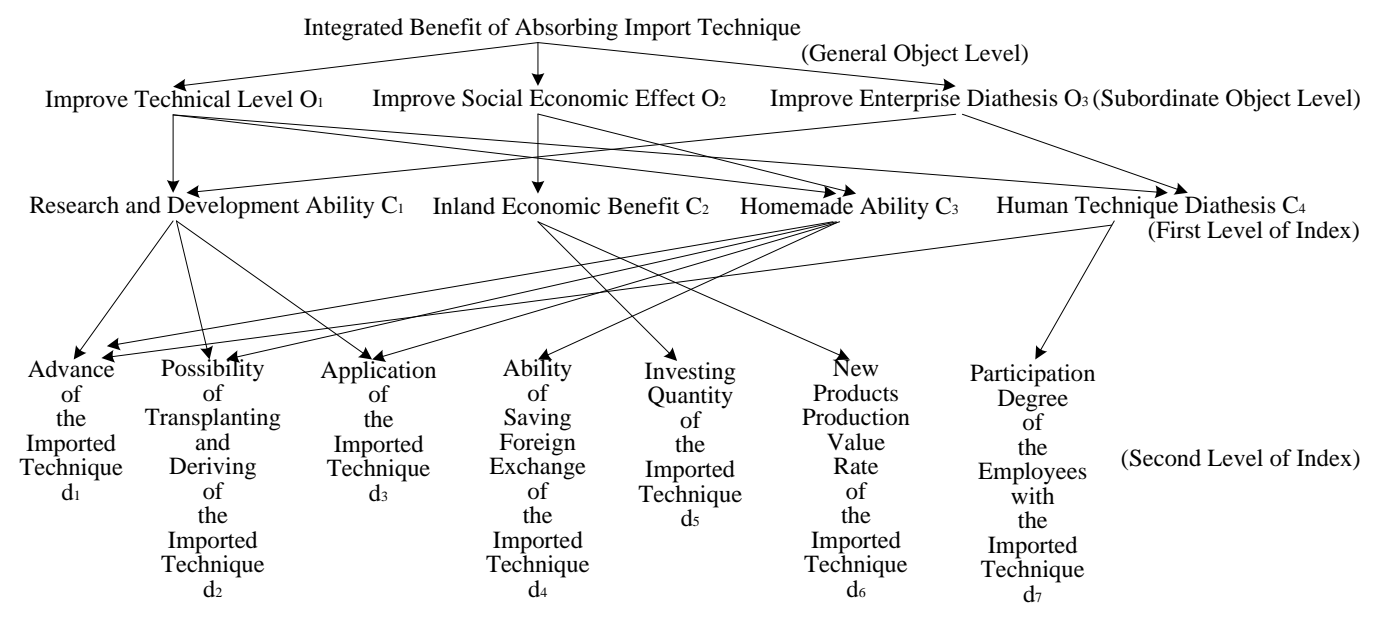

Figure 3 Latter stage evaluation index system of absorbing import project

\section{Case Analysis}

This paper considers that synthetic benefits of technique import project's digest and absorbing mainly put up the contribution degree of project's digest and absorbing to technique import target. Hereby it is the total target of technique import project's digest and absorbing now that: improving the electric power technology ability through importing, digesting and absorbing the foreign advanced technique, then promoting the national economic development ( $\mathrm{T})$. It is the centralized manifest of synthetic benefits of digesting and absorbing project. The corresponding target is: raising the independent technique level $\left(\mathrm{O}_{1}\right)$, improving social economic effect $\left(\mathrm{O}_{2}\right)$, and improving the enterprise quality $\left(\mathrm{O}_{3}\right)$. Through decomposition of import object by level, we can obtain the evaluation index system framework of project's digest and absorbing effect (Figure 3).

The main characters of this evaluation index system are:

(1) Decompose the demand of total target gradually to the idiographic import project by taking the object system of technique import, digest and absorbing as the backing. So it is systemic.

(2) According to the index connotation size and the relationship between them, the index is arranged as multilevel. So it is obvious hierarchical.

(3) Each index is a multi-information carrier in ordinary circumstances. Here it formed the mixed-type structure because of actual condition, overcame the weakness that the complete independent structure possibly brings information loss in system evaluation. So it is scientific.

(4) When choosing the evaluation index, we should consider the actuality of the electrical power office as much as possible. Then it is possible for the index system evaluation.

The electrical power office imported some manufacture technique of power transformer equipment in the period of the "eighth five-year plan". Some power transformer equipment manufacture enterprise imported six techniques; three of them normally went into production one after another. We should keep these three projects secret, so we call them ABC import project

According to the established system (comprising index weight) and certain method of system evaluation, first of all, basically find out and approve all the firsthand information and related data which is demanded by the basic

(to be continued on Page 80) 